\title{
OPEN Lymph node metastasis around the entrance point to recurrent laryngeal nerve in papillary thyroid carcinoma
}

\author{
Tian $\mathrm{Lv}^{1,2}$, Zhuoran Liu' ${ }^{1,2}$ \& JiqiYan ${ }^{1 *}$ \\ There are few reports on the lymph nodes of entrance point to recurrent laryngeal nerve (LN-epRLN) \\ in patients with papillary thyroid carcinoma (PTC). Thus, we investigated the clinical significance of \\ LN-epRLN and implications it may have. An observational analysis of 878 consecutive patients with \\ PTC who underwent thyroidectomy from April 2016 to March 2017 was conducted. We explored the \\ surrounding tissue of laryngeal entry point, during routine central lymph node dissection (CLND). The \\ lymph node specimens were sent separately for routine histopathological examination. Thereafter, \\ complications and follow-ups were recorded. LN-epRLNs were found in 73 of the 878 patients, with the \\ metastatic rate of $3.76 \%$. Univariate and multivariate analysis showed central lymph node metastases \\ can serve as independent predictors for LN-epRLN metastasis. In summary, we confirmed the \\ significance of $L N$-epRLN in metastasis and recurrence, which required precise anatomy and thorough \\ CLND. In PTC patients, especially in suspicious presence of central cervical lymph node metastasis, \\ attention should be given to excising the nodal tissue at the laryngeal entry point.
}

Papillary thyroid carcinoma (PTC) is the most common type of endocrine malignancy yet ${ }^{1}$. Advocates of partial central lymph node dissection (CLND) cite the reduction in risks of postoperative complications ${ }^{2}$. Taken collectively, studies ${ }^{3-5}$ showed that the most common metastases and local recurrences of PTC nodal disease were in the central neck compartment. Additionally, recurrent malignancy after partial CLND may require a second surgery, which is considered more hazardous than a primary operation.

Reoperation on patients with regional recurrence at the laryngeal entry point would pose challenges due to its complexity ${ }^{6}$. Variations in the extent of adhesions around the lymph nodes of entrance point to recurrent laryngeal nerve (LN-epRLN)and neck muscle remnants make identification of the recurrent laryngeal nerve (RLN) and parathyroid glands more difficult compared to primary operation, and risks of certain postoperative complications, such as RLN palsy or hypoparathyroidism, are significantly higher ${ }^{7}$.

In this study, we evaluated a series of patients who underwent thyroidectomies with the aim of highlighting LN-epRLN metastasis in PTC patients.

\section{Methods}

A total of 878 consecutive PTC patients received initial thyroidectomy at Shanghai Ruijin Hospital between April 2016 and March 2017. A prophylactic or therapeutic CLND was routinely performed in PTC patients. Exclusion criteria: those who underwent previous thyroid surgery; possess pathological types of thyroid carcinomas other than PTC; gross extrathyroidal extension invading the surrounding tissue of entrance point to RLN. According to the guidance of the 2018 Tumour Node Metastasis (TNM) staging system of American Joint Committee on Cancer/International Union Against Cancer (AJCC/UICC) ${ }^{8}$, we identified all the sample as $\mathrm{T}_{1-3} \mathrm{~N}_{0-1 \mathrm{a}} \mathrm{M}_{0}$ PTC. All patients involved in this study gave their informed consent, and the data were anoymized. The study was approved by the medical ethics review committee in Shanghai Ruijin Hospital.

All operations in our study were performed by the same group of surgeon (JQ Y.). Central lymph nodes included the Delphian, pretracheal, and paratracheal lymph nodes (LNs). We defined that LN-epRLN as lymph adipose tissue within $5 \mathrm{~mm}$ from the outer edge of the lymph node to the RLN entrance point. Steps for dissection 


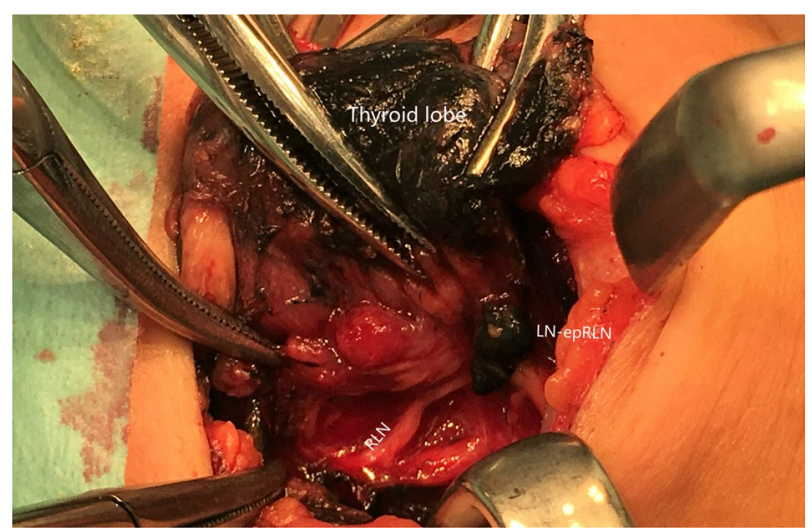

Figure 1. LN-epRLN area coverage. LN-epRLN, lymph node located within the distance from outer edge of the lymph node to RLN entrance point less than $5 \mathrm{~mm}$.

\begin{tabular}{|c|c|c|c|c|}
\hline & & $\begin{array}{l}\text { LN-epRLN } \\
(+)\end{array}$ & $\begin{array}{l}\text { LN-epRLN } \\
(-)\end{array}$ & P-value ${ }^{d}$ \\
\hline \multicolumn{2}{|c|}{ Total (patients) } & 33 & 40 & \\
\hline Age(years) & & $34.20 \pm 10.34$ & $40.00 \pm 10.25$ & 0.118 \\
\hline \multirow{2}{*}{ Gender } & Male & $9(27.3 \%)$ & $5(12.5 \%)$ & \multirow{2}{*}{0.114} \\
\hline & Female & $24(72.7 \%)$ & $35(87.5 \%)$ & \\
\hline \multirow{2}{*}{$\begin{array}{l}\text { TgAb and } \\
\text { TPOAb } \\
\text { levels }\end{array}$} & Elevated & $18(54.5 \%)$ & $20(50.0 \%)$ & \multirow{2}{*}{0.704} \\
\hline & Normal & $15(45.5 \%)$ & $20(50.0 \%)$ & \\
\hline \multirow{2}{*}{$\begin{array}{l}\text { Other } \\
\text { Lymph node } \\
\text { metastases }^{c}\end{array}$} & $(+)$ & $31(93.9 \%)$ & $17(42.5 \%)$ & \multirow{2}{*}{0.000} \\
\hline & $(-)$ & $2(6.1 \%)$ & $23(57.5 \%)$ & \\
\hline
\end{tabular}

Table 1. Univariate analysis showing clinical features associated with risk factors for LN-epRLN ${ }^{a}$ metastasis in the PTC patients ${ }^{\mathrm{b}} .{ }^{\mathrm{a}} \mathrm{LN}$-epRLN: lymph nodes of entrance point to recurrent laryngeal nerve. ${ }^{\mathrm{b}} \mathrm{LN}$-epRLN was found in 73 of the 878 patients. 'Other Lymph node means the central lymph nodes, LN-epRLN excepted. ${ }^{\mathrm{d} P}$ value of $<0.05$ was considered to represent statistical significance.

of central LNs were as follows. Dissection of the lymph nodes around thyroid was completed at the same time as thyroidectomy. After thyroidectomy, the full length of RLN in the neck was revealed, and paratracheal lymph nodes (including LN-epRLN) were removed. All the surgical operations were performed in accordance with the relevant protocols and regulations. During the operation, Using a $1 \mathrm{ml}$ syringe and a 27 -gauge needle, approximately $0.1 \mathrm{ml}$ of Carbon nanoparticles (Chongqing Lummy Pharmaceutical Co. Ltd., China) was slowly injected into the lobe. With gentle pressure applied, the surrounding lymph tissue, and the central-compartment LNs could be fully imaged in black 9 . The parathyroid glands were found to be visibly different from the thyroid glands and the LN tissues ${ }^{10}$ (Fig. 1).

All specimens of thyroid lesions were confirmed to be PTCs by postoperative pathological examination. The pre-operative and postoperative clinicopathological features were recorded, which included the following variables: age, gender, TgAb and TPOAb levels, primary tumor size and location, LN-epRLN laterality, thyroid nodules in primary lobe, and lymph node metastasis based on the final pathology report. 73 cases all had completed follow-up data (follow-up period from 24 to 36 months). The present study included the postoperative US examination and procedure-related complications.

Statistical analysis. To identify differences between groups for specific variables, SPSS version 16 software (SPSS Inc, Chicago, IL) was used for statistical analysis. Univariate analysis was performed using Chi-square test. A $P$-value of $<0.05$ was considered to represent statistical significance.

\section{Results}

In our experience, there were two metastatic lesions in the central compartment and the two patients suffered a second operation, which both resulted in the overlooked LN-epRLN. Our observational study of 878 consecutive patients with PTC was inspired by that.

LN-epRLNs were found in 73 of all the 878 patients (8.31\%) and the metastasis rate was $3.76 \%(33 / 878)$. There were no significant difference in gender $(P=0.114)$ and age $(P=0.118)$ between the two groups (Table 1$)$. For PTC patients presenting no LN-epRLN, 48.2\% (388/805) had central LN metastatic rate, while 65.8\% (48/73) PTC patients presenting LN-epRLNs had central LN metastases. Other central nodal metastases were more common in the positive LN-epRLN group ( 93.9 vs. $42.5 \%, P<0.001)$. 38 patients were found to have elevated $\mathrm{TgAb}$ or TPOAb level, indicative of Hashimoto's thyroiditis, among them, 15 patients (54.5\%) had positive LN-epRLN. 


\begin{tabular}{|c|c|c|c|c|}
\hline & $\begin{array}{l}\text { LN- } \\
\text { epRLN(+) }\end{array}$ & $\begin{array}{l}\text { LN- } \\
\text { epRLN(-) }\end{array}$ & P-value \\
\hline \multicolumn{2}{|c|}{$\begin{array}{l}\text { Total (involved thyroid } \\
\text { lobes)* }\end{array}$} & 37 & 42 & \\
\hline \multirow{2}{*}{ Laterality } & Left & $16(43.2 \%)$ & $22(52.4 \%)$ & \multirow{2}{*}{0.424} \\
\hline & Right & $21(56.8 \%)$ & $20(47.6 \%)$ & \\
\hline \multirow{2}{*}{ Multifocality ${ }^{\mathrm{d}}$} & $(+)$ & $25(67.6 \%)$ & $14(33.3 \%)$ & \multirow{2}{*}{0.033} \\
\hline & $(-)$ & $12(32.4 \%)$ & $28(66.7 \%)$ & \\
\hline \multirow{3}{*}{ Tumor Size } & $<10 \mathrm{~mm}$ & $9(24.3 \%)$ & $17(40.5 \%)$ & \multirow{3}{*}{0.288} \\
\hline & $\begin{array}{l}10- \\
20 \mathrm{~mm}\end{array}$ & $15(40.5 \%)$ & $15(35.7 \%)$ & \\
\hline & $>20 \mathrm{~mm}$ & $13(35.1 \%)$ & $10(23.8 \%)$ & \\
\hline \multirow{2}{*}{ Tumor Location } & upper $1 / 3$ & $25(67.6 \%)$ & $15(35.7 \%)$ & \multirow{2}{*}{0.044} \\
\hline & lower $2 / 3$ & $12(32.4 \%)$ & $27(64.3 \%)$ & \\
\hline
\end{tabular}

Table 2. Univariate analysis showing clinical features associated with risk factors for LN-epRLN ${ }^{a}$ metastasis in the involved PTC thyroid lobes ${ }^{\mathrm{b}}$. ${ }^{\mathrm{L}} \mathrm{LN}$-epRLN: lymph nodes of entrance point to recurrent laryngeal nerve. ${ }^{\mathrm{b}} \mathrm{LN}$ epRLN was identified bilaterally in 6 of the 73 patients. Thus, there was 79 involved thyroid lobes in total. ${ }^{c}$ value of $<0.05$ was considered to represent statistical significance. ${ }^{\mathrm{d}}$ Defined as more than one PTC in the same lobe as the primary carcinoma by the postoperative pathology.

\begin{tabular}{|l|l|l|}
\hline & OR (95\% CI) & P-value $^{\mathbf{c}}$ \\
\hline Laterality (Left vs Right) & $0.511(0.209-1.254)$ & 0.178 \\
\hline Multifocality & $1.750(0.717-4.272)$ & 0.057 \\
\hline $\begin{array}{l}\text { Tumor Size }(<20 \mathrm{~mm} \\
\text { vs }>20 \mathrm{~mm})\end{array}$ & $1.733(0.651-4.617)$ & 0.325 \\
\hline $\begin{array}{l}\text { Tumor Location (upper } \\
1 / 3 \text { vs lower 2/3) }\end{array}$ & $1.957(0.797-4.784)$ & 0.046 \\
\hline $\begin{array}{l}\text { Other Lymph node } \\
\text { metastases }^{\mathrm{e}}\end{array}$ & $20.971(4.401-99.924)$ & 0.000 \\
\hline
\end{tabular}

Table 3. Multivariate analysis showing clinical features associated with risk factors for LN-epRLN ${ }^{a}$ metastasis in the involved PTC thyroid lobes ${ }^{\mathrm{b}}$. ${ }^{\mathrm{L}} \mathrm{LN}-\mathrm{epRLN}$ : lymph nodes of entrance point to recurrent laryngeal nerve. ${ }^{b} \mathrm{LN}$-epRLN was identified bilaterally in 6 of the 73 patients. Thus, there was 79 involved thyroid lobes in total. ${ }^{c} \mathrm{P}$-value of $<0.05$ was considered to represent statistical significance. ${ }^{\mathrm{d}}$ Defined as more than one PTC in the same lobe as the primary carcinoma by the postoperative pathology. ${ }^{e}$ Other Lymph node means the central lymph nodes, LN-epRLN excepted.

However, no significant relation was found between LN-epRLN metastasis and serum TgAb and TPOAb levels $(\mathrm{P}=0.704)$ (Table 1$)$.

LN-epRLN was identified bilaterally in 6 patients. Thus, there were 79 involved thyroid lobes in total. In this present study, we found that there were variant nodes of 1 to 4 (1.51 on average), with a positive rate of $46.84 \%$ (37/79). Then we analyzed the risk factors for LN-epRLN metastasis in the involved PTC thyroid lobes. The incidence of right LN-epRLN was higher than left (Right 41 vs Left 38). In univariate analysis, tumor located in the upper third $(P=0.044)$ and tumor multifocality $(P=0.033)$ were significant (Table 2$)$. In our study, no significant relation to LN-epRLN metastasis was found with tumor size $(P=0.288)$.

Further, multivariate analysis (Table 3 ) displayed that tumor location $(\mathrm{OR}=1.957,95 \% \mathrm{CI}$ : 0.797-4.784, $P<0.05)$ and other central nodal metastases (OR $=20.971,95 \% \mathrm{CI}$ : $4.401-99.924, P<0.001)$ may be independent risk factors. In line with multivariate analysis, central LN metastasis was stable parameters pointing toward positive LN-epRLN (Fig. 2).

No patient had permanent RLN injury or permanent hypoparathyroidism in this study, and no patient had a second operation during the follow-up period. Seven $(0.8 \%)$ patients had transient hoarseness (4-6 weeks), which were checked under fiberlaryngoscopy at one month and 6 months after surgery. Six (0.7\%) patients had postoperative transient hypoparathyroidism (5-14 days).

\section{Discussion}

Previous literatures ${ }^{3-5}$ have shown that LN involvement is relevant to local recurrence. Because of the increased risk of recurrence with focal "berry-pickin" techniques, compartmental surgery is recommended in accordance to the current American Thyroid Association (ATA) guidelines ${ }^{11,12}$. Our study was inspired by two cases where the PTC patients suffered hoarseness after a second operation addressin the presence of metastatic LN-epRLN, which may have been reserved in consideration of RLN injury and hypoparathyroidism during the primary operation.

The anatomic boundaries of CLND are from the hyoid bone to the innominate artery. An appropriate range of CLND should both reduce the local recurrence rate and the incidence of post-operative complications ${ }^{11}$. In CLND, fear of RLN injury and hypoparathyroidism may lead to insufficient exposure where metastases are left 

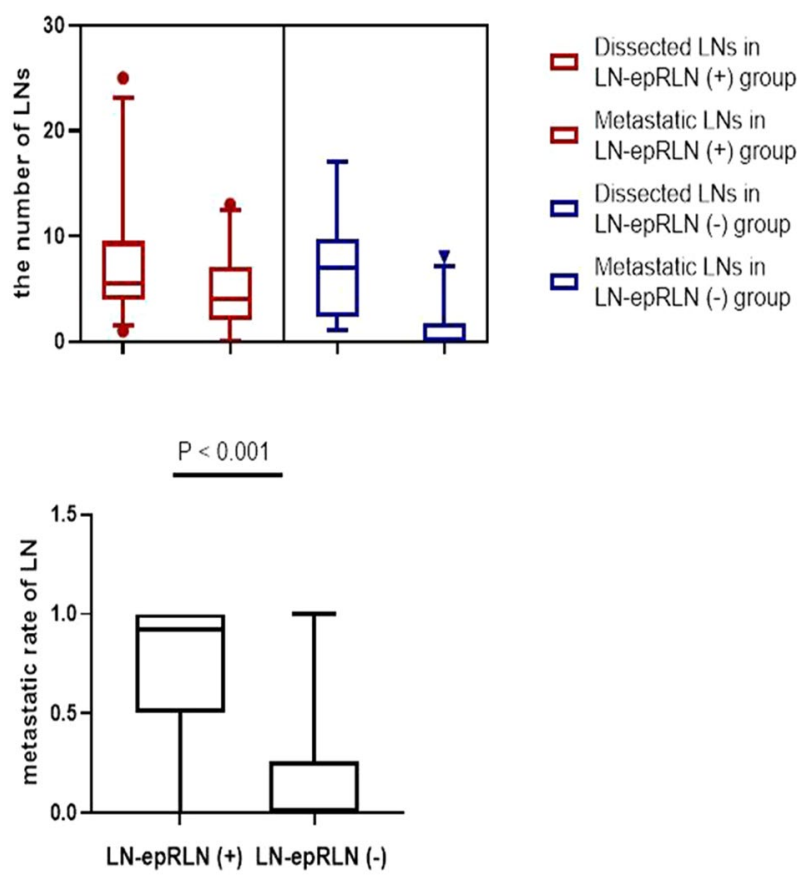

Figure 2. To analyze disease burden of PTC patients, the number of dissected and metastatic LNs were recorded. In our study, central LN metastasis was stable parameters pointing toward positive LN-epRLN.

at the laryngeal entry point. We refer to these as involved LN-epRLNs in our study. To this date, no studies have covered the significance of LN-epRLN, as part of central LNs ${ }^{13,14}$.

LN-epRLN was defined as lymph adipose tissue within $5 \mathrm{~mm}$ from the outer edge of the lymph node to the RLN entrance point (Fig. 1). In our study, there were variant nodes, 1 to 4 (1.51 on average), in diameter between $6 \mathrm{~mm}$ and $10 \mathrm{~mm}$. In the present study, LN-epRLN was found in 73 of 878 patients $(8.31 \%)$ and the metastatic rate was $3.76 \%$ (33/878). After the Carbon nanoparticles mapping, the non-black-stained parathyroid glands were easily discriminated from the lymph-fatty tissue ${ }^{9,10}$. Our findings indicated that CLND could be performed safely with the use of intraoperative $\mathrm{CN}$ mapping, Which has been reported in the previous study ${ }^{10}$.

In terms of involved thyroid lobes, the positive LN-epRLN rate was $46.84 \%$ (37/79), and the incidence of the right side was slightly higher than that on the left side. The left and right RLNs have slight differences in anatomical position, and the cervical part of the esophagus located closely next to the left. Therefore, there was a triangular space located posterior to the right RLN, which makes space for lymph adipose tissue ${ }^{15-17}$. In the literatures, the presence of Hashimoto's thyroiditis leads to reactive lymphaden proliferation ${ }^{18,19}$. We preferred that the patients of Hashimoto's thyroiditis would have positive LN-epRLNs. However, it showed no statistical significance between LN-epRLN metastasis and serum TgAb/TPOAb levels in our study, limited by small samples.

The univariate analysis showed that the factors influencing LN-epRLN involvement were the other central LN metastases, the upper third tumor location and tumor multifocality. Previous studies ${ }^{20-24}$ have found that ipsilateral multifocal disease could be used to predict neck lymph node metastases, which may reflect the ability of clonal formation of cancer cells ${ }^{25-28}$. This result strengthens the argument for CLND (LN-epRLN included) in PTC as preoperative US and intra-operative frozen biopsy shows multifocality of the PTC nodules. In our study, the rate of other central LN metastasis in the presence of LN-epRLN metastasis was $93.9 \%$. In line with multivariate analysis, central LN metastasis was stable parameters pointing toward positive LN-epRLN (Fig. 2), which can be evaluated through preoperative US and frozen biopsy performed intra-operatively.

Usually, most patients with PTC obtain a 10 -year survival rate of $80-90 \%$, but the regional recurrence rate after surgery is $5-20 \%{ }^{12}$. Therefore, it's important to improve the thoroughness of CLND. Our results mirror the data from previous literatures: reoperative CLND has been shown to have rates of RLN injury $21 \%$ transiently and postoperative hypocalcemia with a large range of $0-24 \%^{29-36}$. However, the incidence of complications varies according to the skill and experience of the surgeon ${ }^{7,37}$.

PTC patients with clinically involved LNs in the central compartment should be managed with a LN-epRLN dissection during CLND, taking into consideration RLN and parathyroid glands which are closely related to the nodal basins. The standard exploration and resection procedures are key factors that impact the relative difficulty of performing secondary surgery.

Our observational study was limited by small samples, but we focused on acknowledgement of LN-epRLN. Moreover, patients with lateral neck lymph node matastases were excluded due to inclusion criteria. These patients may have experienced worse outcomes than patients enrolled in our study. However, to our knowledge, this is the first article in English to progress our understanding of LN-epRLN metastases in PTC patients. 


\section{Conclusions}

In summary, we confirmed the significance of LN-epRLN in metastasis and recurrence, which required precise anatomy and thorough CLND. In PTC patients, especially in suspicious presence of central cervical lymph node metastasis, attention should be given to excising the nodal tissue at the laryngeal entry point.

Received: 16 July 2019; Accepted: 6 March 2020;

Published online: 25 March 2020

\section{References}

1. Siegel, R. L., Miller, K. D. \& Jemal, A. Cancer statistics. 2017. CA Cancer J. Clin. 67, 7-30 (2017).

2. Rago, T. et al. Male sex, single nodularity, and young age are associated with the risk of finding a papillary thyroid cancer on fineneedle aspiration cytology in a large series of patients with nodular thyroid disease. Eur. J. Endocrinol. 162, 763-770 (2010).

3. Lundgren, C. I., Hall, P., Dickman, P. W. \& Zedenius, J. Clinically significant prognostic factors for differentiated thyroid carcinoma: a population-based, nested case-control study. Cancer. 106, 524-531 (2006).

4. Rotstein, L. The role of lymphadenectomy in the management of papillary carcinoma of the thyroid. J. Surg. Oncol. 99, 186-188 (2009).

5. Sakorafas, G. H., Sampanis, D. \& Safioleas, M. Cervical lymph node dissection in papillary thyroid cancer: current trends, persisting controversies, and unclarified uncertainties. Surg. Oncol. 19, e57-e70 (2010).

6. Salari, B., Ren, Y., Kamani, D. \& Randolph, G. W. Revision neural monitored surgery for recurrent thyroid cancer: Safety and thyroglobulin response. Laryngoscope. 126, 1020-1025 (2016).

7. Tunca, F. et al. The preoperative exclusion of thyroid carcinoma in multinodular goiter: dynamic contrast-enhanced magnetic resonance imaging versus ultrasonography-guided fine-needle aspiration biopsy. Surgery. 142, 992-1002 (2007).

8. Amin, M. B. et al. AJCC Cancer Staging Manual (8th edn) 1726-1727 (Springer: New York, 2017)

9. Cheng, X. et al. Intraoperative carbon nanoparticles mapping in secondary total thyroidectomy for recurrent thyroid nodules: Results of a 8-criterion case-match study (case control study). Int. J. Surg. 60, 210-215 (2018).

10. Dzodic, R. \& Santrac, N. In situ preservation of parathyroid glands: advanced surgical tips for prevention of permanent hypoparathyroidism in thyroid surgery. J. BUON. 22, 853-855 (2017).

11. Roman, S. A., Sosa, J. A., \& Solórzano, C. C. Management of Thyroid Nodules and Differentiated Thyroid Cancer, 23-45 (Springer International Publishing, 2017).

12. Haugen, B. R. et al. 2015 American Thyroid Association management guidelines for adult patients with thyroid nodules and differentiated thyroid cancer: the American Thyroid Association guidelines task force on thyroid nodules and differentiated thyroid cancer. Thyroid. 26, 1-133 (2016).

13. Chai, Y. J. et al. Papillary thyroid carcinoma located in the isthmus or upper third is associated with Delphian lymph node metastasis. World J. Surg. 38, 1306-1311 (2014).

14. Sun, G. et al. Lymph node metastasis between sternocleidomastoid and sternohyoid muscle in clinically node-positive papillary thyroid carcinoma. Head Neck. 35, 1168-1170 (2013).

15. Kim, Y. S. \& Park, W. C. Clinical predictors of right upper paraesophageal lymph node metastasis from papillary thyroid carcinoma. World J. Surg. Oncol. 10, 164 (2012).

16. Bae, S. Y. et al. Right paraesophageal lymph node dissection in papillary thyroid carcinoma. Ann Surg. Oncol. 19, 996-1000 (2012).

17. Ito, Y. et al. Incidence and predictors of right paraesophageal lymph node metastasis of N0 papillary thyroid carcinoma located in the right lobe. Endocr. J. 60, 389-392 (2013).

18. Liang, J. et al. Clinical analysis of Hashimoto thyroiditis coexistent with papillary thyroid cancer in 1392 patients. Acta Otorhinolaryngol. Ital. 37, 393-400 (2017).

19. Cordioli, M. I. et al. Study of the histological profile of papillary thyroid carcinomas associated with Hashimoto's thyroiditis. Arq Bras Endocrinol. Metabol. 57, 445-449 (2013).

20. Pasieka, J. L., Thompson, N. W., McLeod, M. K., Burney, R. E. \& Macha, M. The incidence of bilateral well-differentiated thyroid cancer found at completion thyroidectomy. World J. Surg. 16, 711-716 (1992).

21. Koo, B. S. et al. Occult contralateral carcinoma in patients with unilateral papillary thyroid microcarcinoma. Ann Surg Oncol. 17, 1101-1105 (2010).

22. Pitt, S. C., Sippel, R. S. \& Chen, H. Contralateral papillary thyroid cancer: does size matter? Am. J. Surg. 197, 342-347 (2009).

23. Hwang, E. et al. Bilateral papillary thyroid cancer and associated histopathologic fndings. J. Otolaryngol Head Neck Surg. 39, 284-287 (2010).

24. Connor, M. P., Wells, D. \& Schmalbach, C. E. Variables predictive of bilateral occult papillary microcarcinoma following total thyroidectomy. Otolaryngol. Head Neck Surg. 144, 210-215 (2011).

25. Shattuck, T. M., Westra, W. H., Ladenson, P. W. \& Arnold, A. Independent clonal origins of distinct tumor foci in multifocal papillary thyroid cancer. N. Engl. J. Med. 352, 2406-2412 (2005).

26. Iida, F., Yonekura, M. \& Miyakawa, M. Study of intraglandular dissemination of thyroid cancer. Cancer. 24, 764-771 (1969).

27. Lee, K. J., Cho, Y. J., Kim, J. G. \& Lee, D. H. How many contralateral papillary thyroid carcinomas can be missed? World J. Surg. 37, 780-785 (2013)

28. Grebe, S. K. \& Hay, I. D. Thyroid cancer nodal metastases: biologic significance and therapeutic considerations. Surg. Oncol. Clin. N. Am. 5, 43-63 (1996).

29. Park, C. H. et al. Significance of the extracapsular spread of metastatic lymph nodes in papillary thyroid carcinoma. Clin. Exp. Otorhinolaryngol. 8, 289-294 (2015).

30. Farrag, T. Y. et al. Algorithm for safe and effective reoperative thyroid bed surgery for recurrent/persistent papillary thyroid carcinoma. Head Neck. 29, 1069-1074 (2007).

31. Onkendi, E. O. et al. Reoperative experience with papillary thyroid cancer. World J. Surg. 38, 645-652 (2014).

32. Shah, M. D. et al. Efficacy and safety of central compartment neck dissection for recurrent thyroid carcinoma. Arch Otolaryngol. Head Neck Surg. 138, 33-37 (2012).

33. Alvarado, R., Sywak, M. S., Delbridge, L. \& Sidhu, S. B. Central lymph node dissection as a secondary procedure for papillary thyroid cancer: is there added morbidity? Surgery. 145, 514-518 (2009).

34. Tufano, R. P., Bishop, J. \& Wu, G. Reoperative central compartment dissection for patients with recurrent/persistent papillary thyroid cancer: efficacy, safety, and the association of the BRAF mutation. Laryngoscope. 122, 1634-1640 (2012).

35. Lang, B. H. et al. Evaluating the morbidity and efficacy of reoperative surgery in the central compartment for persistent/recurrent papillary thyroid carcinoma. World J. Surg. 37, 2853-2859 (2013).

36. Shen, W. T. et al. Central neck lymph node dissection for papillary thyroid cancer: comparison of complication and recurrence rates in 295 initial dissections and reoperations. Arch Surg. 145, 272-275 (2010).

37. Amin, M. B. et al. The Eighth Edition AJCC Cancer Staging Manual: continuing to build a bridge from a population-based to a more "personalized" approach to cancer staging, CA Cancer. J. Clin. 67, 93-99 (2017). 


\section{Acknowledgements}

Address all correspondence and requests for reprints to: Jiqi Yan, Department of General Surgery, Ruijin Hospital, Shanghai Jiao Tong University School of Medicine, No.197 Ruijin er Road, Shanghai 200025, China. E-mail: yanjiqi2000@163.com.

\section{Author contributions}

T.L. recorded the data and drafted the manuscript. Z.L. participated in the design of the study and performed the statistical analysis. J.Y. conceived of the study, and participated in its design and coordination and helped to draft the manuscript. All authors read and approved the final manuscript.

\section{Competing interests}

The authors declare no competing interests.

\section{Additional information}

Correspondence and requests for materials should be addressed to J.Y.

Reprints and permissions information is available at www.nature.com/reprints.

Publisher's note Springer Nature remains neutral with regard to jurisdictional claims in published maps and institutional affiliations.

Open Access This article is licensed under a Creative Commons Attribution 4.0 International License, which permits use, sharing, adaptation, distribution and reproduction in any medium or format, as long as you give appropriate credit to the original author(s) and the source, provide a link to the Creative Commons license, and indicate if changes were made. The images or other third party material in this article are included in the article's Creative Commons license, unless indicated otherwise in a credit line to the material. If material is not included in the article's Creative Commons license and your intended use is not permitted by statutory regulation or exceeds the permitted use, you will need to obtain permission directly from the copyright holder. To view a copy of this license, visit http://creativecommons.org/licenses/by/4.0/.

(c) The Author(s) 2020 